\title{
Dynamic behavior of chemical reactivity indices in density functional theory: A Bohn-Oppenheimer quantum molecular dynamics study
}

\author{
SHUBIN LIU \\ Department of Academic Technology \& Networks, University of North Carolina, Chapel Hill, \\ North Carolina 27599-3455, USA \\ e-mail: shubin@email.unc.edu
}

\begin{abstract}
Dynamic behaviors of chemical concepts in density functional theory such as frontier orbitals (HOMO/LUMO), chemical potential, hardness, and electrophilicity index have been investigated in this work in the context of Bohn-Oppenheimer quantum molecular dynamics in association with molecular conformation changes. Exemplary molecular systems like $\mathrm{CH}_{5}^{+}, \mathrm{Cl}^{-}\left(\mathrm{H}_{2} \mathrm{O}\right)_{30}$ and $\mathrm{Ca}^{2+}\left(\mathrm{H}_{2} \mathrm{O}\right)_{15}$ are studied at $300 \mathrm{~K}$ in the gas phase, demonstrating that HOMO is more dynamic than LUMO, chemical potential and hardness often fluctuate concurrently. It is argued that DFT concepts and indices may serve as a good framework to understand molecular conformation changes as well as other dynamic phenomena.
\end{abstract}

Keywords. Chemical potential; hardness; electrophilicity index; dynamics; HOMO; LUMO; QMD.

\section{Introduction}

Chemical concepts, reactivity indices and rules, ${ }^{1,2}$ stemming from density functional theory (DFT), such as frontier orbitals (HOMO/LUMO), electronegativity (chemical potential), global and local hardness and softness, Fukui function, electrophilicity index, hard and soft acid and base (HSAB), maximum hardness principle (MHP) etc., have attracted considerable interests in the literature in the last few decades. Applications of these concepts have been made to understand chemical reactivity such as electrophilicity, ${ }^{3}$ aromaticity, ${ }^{4}$ transition state, ${ }^{5}$ etc. Almost all these works have been based on the time-independent DFT framework. There has been relatively scarce study of the time evolution of the DFT based concepts, reactivity indices and principles. To the best knowledge of the present author, the main work along that line comes from Chattaraj and co-workers ${ }^{6-12}$ within quantum fluid DFT, where a generalized nonlinear Schrödinger equation is solved numerically. Another work is by Vuilleumier and Sprik ${ }^{13}$ who investigated the electronic structure of both hard and soft ions $\left(\mathrm{Na}^{+}\right.$and $\mathrm{Ag}^{+}$respectively) in aqueous solution using Car-Parinello molecular dynamics. ${ }^{14}$ It is also worth mentioning the relevant work by York and Yang ${ }^{15}$ on the dynamical behavior of Fukui functions via a chemical potential equalization method.
In this report, we make use of the Bohn-Oppenheimer quantum molecular dynamics scheme, where the potential energy/derivatives of a system are treated purely quantum mechanically and the nuclei move on the electronic potential energy surface via the Newton's classical equation of motion, and apply it to three exemplary molecular systems to observe the time evolution of frontier orbitals, chemical potential, global hardness, and electrophilicity index. The purpose of this preliminary study is to couple the conformation change of molecular systems with the time evolution of chemically important DFT concepts and indices and demonstrate the correlation between them and thus the usefulness of DFT concepts and indices in understanding the process of molecular conformation changes as well as other dynamic phenomena.

\section{Methodology}

It is well known ${ }^{19}$ that chemical potential, $\mu$, and the electronegativity, $\chi$, could be written as the partial derivative of the system's energy with respect to the number of electrons $N$ at the fixed external potential $v(\boldsymbol{r})$ :

$$
\mu=-\chi=-(\partial E / \partial N)_{v(\mathbf{r})} .
$$

and the global chemical hardness $\eta$ is defined as 

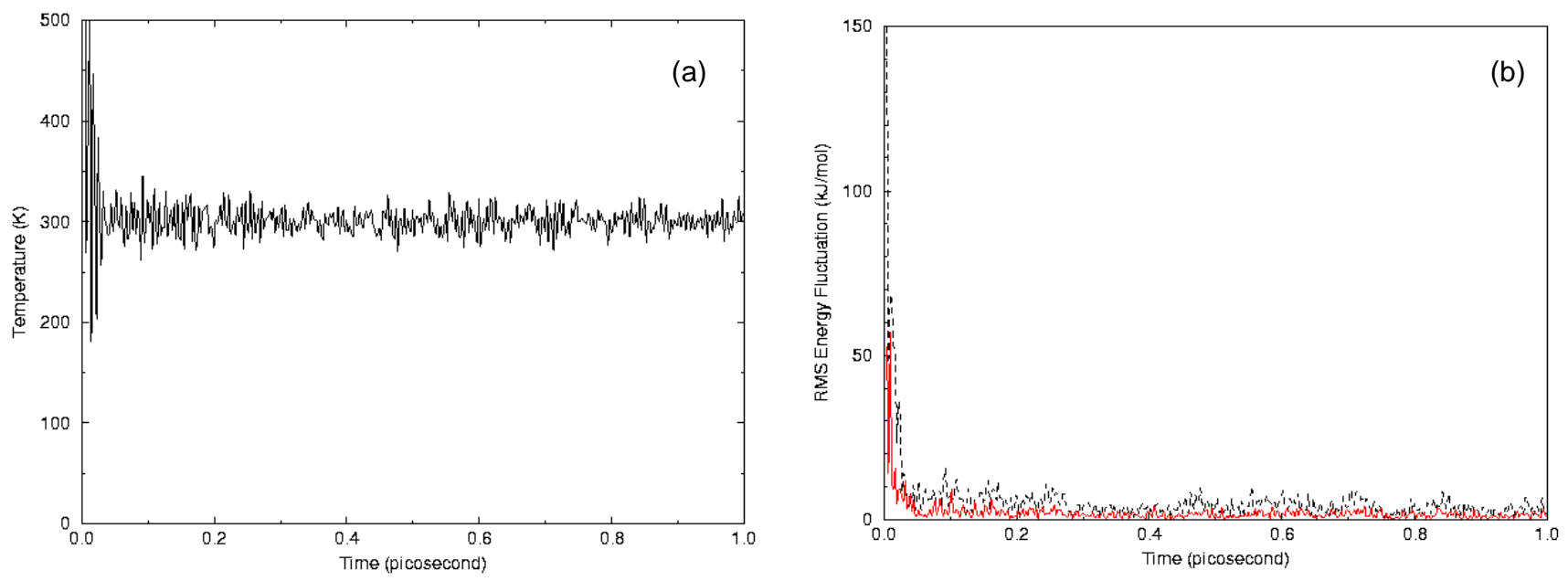

Figure 1. (a) Fluctuations of the simulation temperature during a QMD simulation run, showing that it quickly reaches equilibrium at $300 \mathrm{~K}$; (b) RMS (root-mean-square) energy fluctuations of the potential energy (black dotted line) and total energy (solid red line) for the chloride anion in 30 water molecules, $\mathrm{Cl}^{-}\left(\mathrm{H}_{2} \mathrm{O}\right)_{30}$.

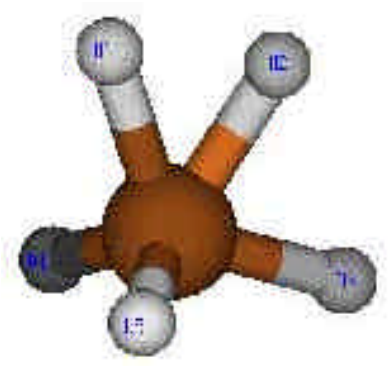

$\mathrm{C}_{\mathrm{s}}(\mathrm{I})$

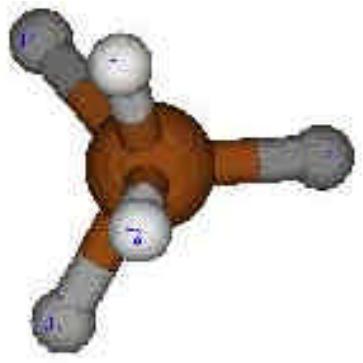

$\mathrm{C}_{\mathrm{s}}(\mathrm{II})$

Figure 2. The two relatively stable conformers of the protonated methane, $\mathrm{CH}_{5}^{+}$, each of which has $C_{s}$ symmetry and can be viewed as $\mathrm{H}_{1}-\mathrm{H}_{2}$ lying above the $\mathrm{CH}_{3}^{+}$plane. The left conformer is energetically more stable than the right conformer.

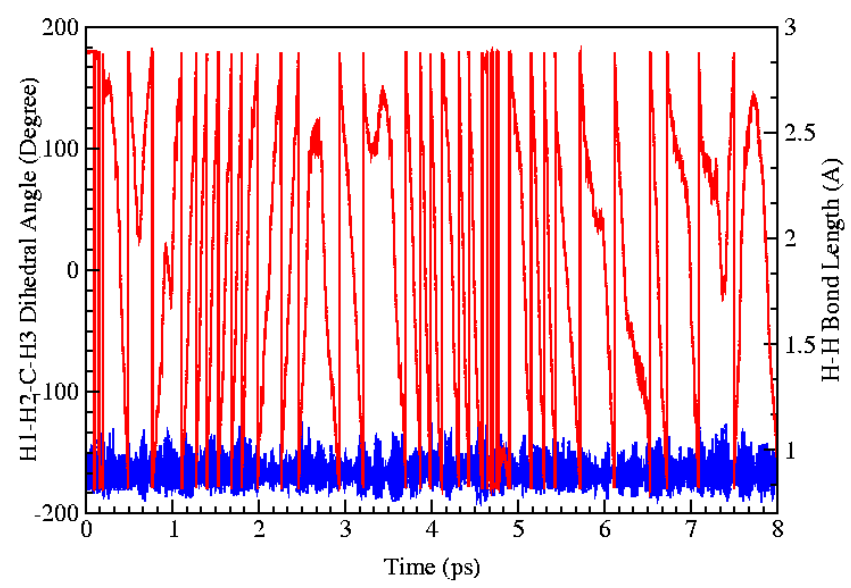

Figure 3. Time profile of the $\mathrm{H}_{2}$ fragment rotation (red) and $\mathrm{H}_{1}-\mathrm{H}_{2}$ bond stretching (blue) over the $\mathrm{CH}_{3}^{+}$plane.

$$
\eta=\frac{1}{2}\left(\partial^{2} E / \partial N^{2}\right)_{\vee(r)}
$$

According to Mulliken, ${ }^{20}$ one has

$$
\mu=-\chi=-\frac{1}{2}(I+A),
$$

and $^{21}$

$$
\eta=\frac{1}{2}(I-A)
$$

where $I$ and $A$ are the first ionization energy and electron affinity, respectively. In the finite different approximation, the ionization energy and electron affinity can be replaced by the HOMO and LUMO energy, respectively, using Koopmans' theorem, ${ }^{22}$ within a Hartree-Fock and/or density functional theory schemes, with $I=-\varepsilon_{\text {номо }}$ and $A=-\varepsilon_{\mathrm{LUMO}}$, yielding

$$
\mu=-\chi=-\frac{1}{2}\left(\varepsilon_{\mathrm{HOMO}}+\varepsilon_{\mathrm{LUMO}}\right)
$$

and

$$
\eta=\frac{1}{2}\left(\varepsilon_{\text {LUMO }}-\varepsilon_{\text {HOMO }}\right) .
$$

Recently, Parr et $a l^{23}$ proposed a new DFT concept called the electrophilicity index, $\omega$, in terms of the above two global activity indices, $\mu$ and $\eta$,

$$
\omega=\mu^{2} / 2 \eta,
$$



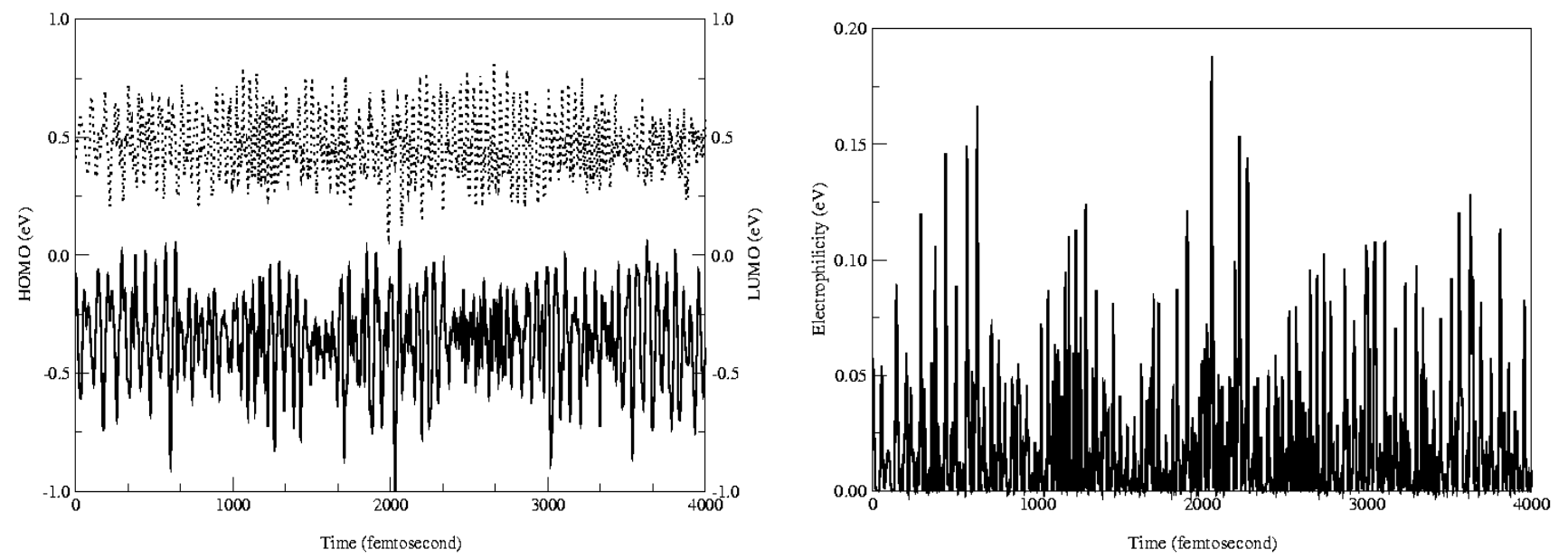

Figure 4. Time profiles of the HOMO (solid line, left) and LUMO (dotted line, left) energies and electrophilicity index (right) for $\mathrm{CH}_{5}^{+}$.

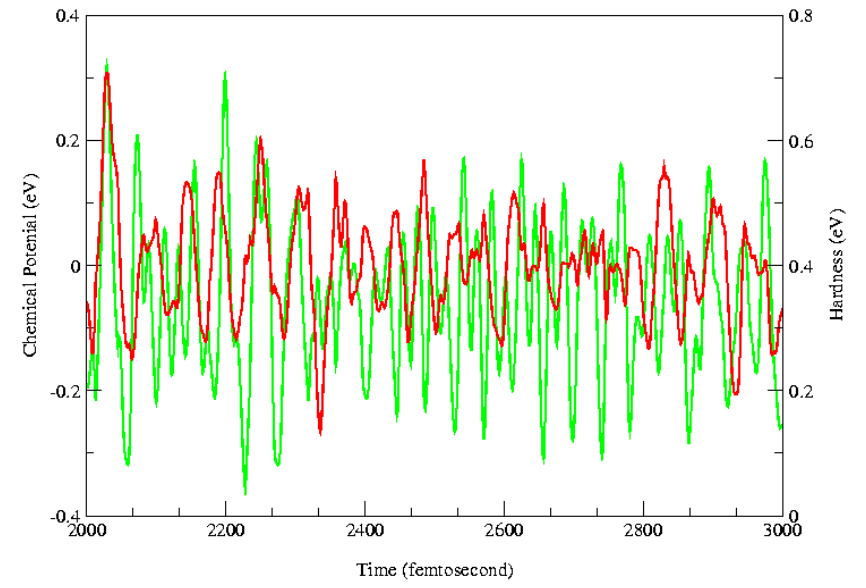

Figure 5. Time evolution profile of chemical potential (green line) and hardness (red line) for the $\mathrm{CH}_{5}^{+}$cation.

which measures the capacity of an electrophile to accept the maximal number (a fractional of or more than one) of electrons in a neighboring reservoir of electron sea. The above three formulas are the working equations for us to calculate the chemical potential, hardness, and electrophilicity index over the course of time evolution.

\section{Computational details}

An extensive review of the Born-Oppenheimer molecular dynamics method is available in the literature. $^{16}$ In the implementation of QMD in the NWChem package, ${ }^{17,18}$ both ab initio and DFT approaches are accessible for the quantum mechanic calculation. As shown here merely as a proof of concept, we use the Hartree-Fock method for two molecular systems, the protonated methane, $\mathrm{CH}_{5}^{+}$, and one chloride anion immersed in 30 water molecules, $\mathrm{Cl}^{-}\left(\mathrm{H}_{2} \mathrm{O}\right)_{30}$, and density functional theory at the B3LYP level for the $\mathrm{Ca}^{2+}\left(\mathrm{H}_{2} \mathrm{O}\right)_{15}$ cluster, a $\mathrm{Ca}^{2+}$ cation in 15 water molecules. We choose these systems because of their relatively flatter potential energy surface and thus richer dynamic phenomena. The basis set employed for the first and last systems is 6$31 \mathrm{G}(\mathrm{d})$ but for the bigger cluster, $\mathrm{Cl}^{-}\left(\mathrm{H}_{2} \mathrm{O}\right)_{30}$, we choose 3-21G instead. The impact of basis set choices has been examined with a smaller system, $\mathrm{Ca}^{2+}\left(\mathrm{H}_{2} \mathrm{O}\right)_{8}$, with three standard basis sets, 3-21G, 6-31G(d) and aug-cc-pVTZ, and no qualitative difference of dynamic results has been found.

The QMD simulation has been performed in the following way. After an initial structure optimization of 120 steps, a system is undergone quantum molecular dynamics simulations under $300 \mathrm{~K}$ for 10 picoseconds with a step size of 0.5 femtosecond and the leapfrog integration algorithm. We employ a constant temperature ensemble using Berendsen's thermostat with the temperature relaxation time set to be 2 fs enabling the system to quickly reach equilibrium (as shown by the temperature fluctuation and potential and total energy's RMS fluctuations in figure 1). The cutoff radius for short range interactions is $2.8 \mathrm{~nm}$. SHAKE is disabled and thus all bonding interactions are treated according to the force calculated from quantum mechanics. QMD output and trajectories are saved in every 5 femtoseconds, and are processed and analyzed aftermaths. Figure 1 illustrates the 

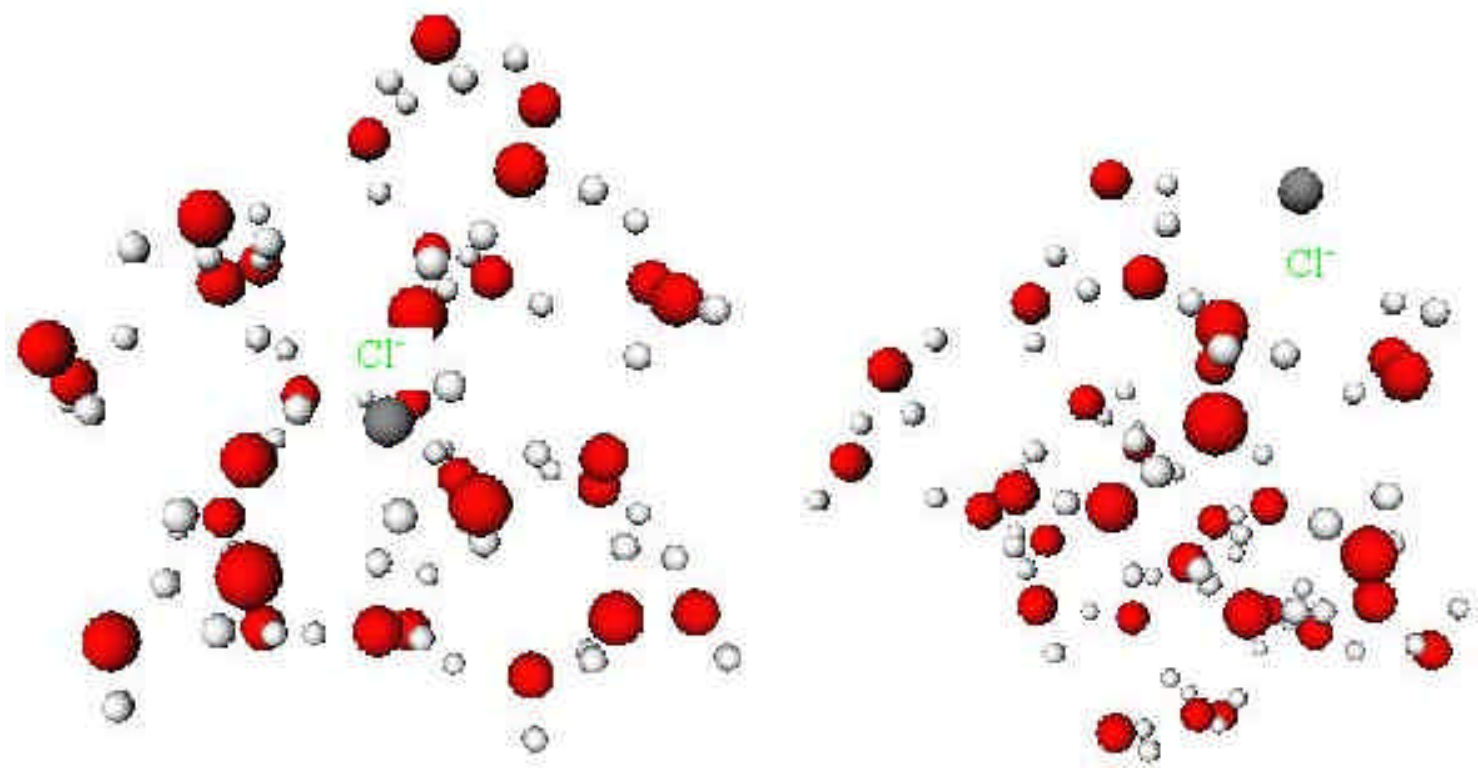

Figure 6. The initial (left) and final (right) conformation of the $\mathrm{Cl}^{-}\left(\mathrm{H}_{2} \mathrm{O}\right)_{30}$ cluster from 15 picosecond QMD simulation.
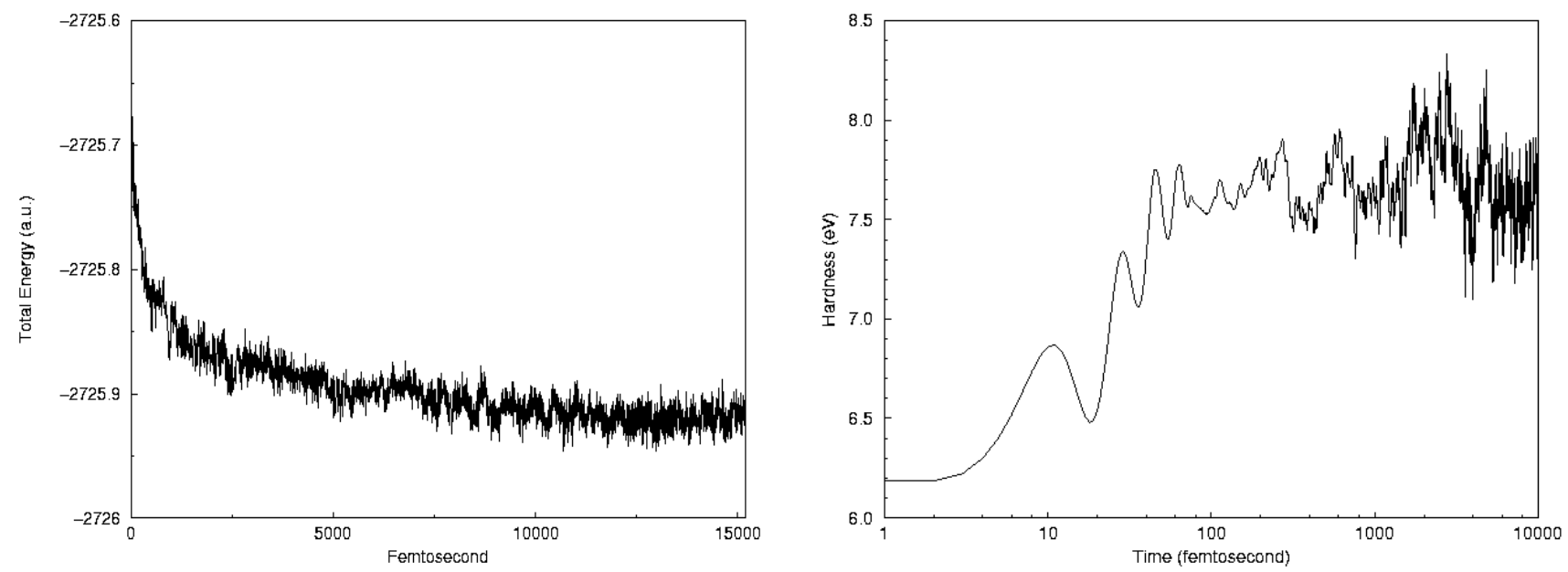

Figure 7. The total energy and hardness profiles of the chloride anion in 30 water molecules, $\mathrm{Cl}^{-}\left(\mathrm{H}_{2} \mathrm{O}\right)_{30}$.

temperature variation and RMS energy fluctuation for the potential and total energies for a portion of a QMD simulation for the chloride anion in 30 water molecules, $\mathrm{Cl}^{-}\left(\mathrm{H}_{2} \mathrm{O}\right)_{30}$, indicating that the system is stable and quickly reaches the equilibrium state.

\section{Results and discussion}

The protonated methane cation, $\mathrm{CH}_{5}^{+}$, is a much controversial species ${ }^{23-27}$ largely because of its very flat potential energy surface and thus no stable conformer has been unambiguously determined either experimen- tally or computationally. We investigate this cation only to demonstrate its rich dynamic behavior and avoid addressing its controversial structure. In figure 2 , two relatively stable conformers are shown, each of which possesses a $C_{s}$ symmetry, with the one on the left, $C_{s}(\mathrm{I})$, a little lower energetically. They can be viewed as a hydrogen molecule $\mathrm{H}_{2}$ floating above the surface of a planar $\mathrm{CH}_{3}^{+}$cation. The difference between the two conformers is that in one case the hydrogen molecule $\mathrm{H}_{1}-\mathrm{H}_{2}$ aligns with the $\mathrm{C}-\mathrm{H}_{3}$ bond whereas in the other case $\mathrm{H}_{1}-\mathrm{H}_{2}$ rotates 90 degrees lining up itself in the direction of $\mathrm{H}_{4}-\mathrm{H}_{5}$. That 
is, one conformer can be viewed to be from a 90degree rotation $\mathrm{H}_{1}-\mathrm{H}_{2}$ of the other. Given that the rotation barrier between the two conformers is small (less than $1 \mathrm{kcal} / \mathrm{mol}$ depending on the level of theory and basis set), it is conceivable that QMD simulations at room temperature should be able to observe the conformation change between them.

This is exactly what we have obtained in QMD simulations at the RHF/6-31G* level. Figure 3 shows the anticipated rotation of the $\mathrm{H}_{1}-\mathrm{H}_{2}$ fragment over the $\mathrm{CH}_{3}^{+}$plane. One finds that the $\mathrm{H}_{1}-\mathrm{H}_{2}-\mathrm{C}-\mathrm{H}_{3}$ dihedral angle alternates between 180 and -180 degrees, while the $\mathrm{H}_{1}-\mathrm{H}_{2}$ bond length keeps relatively unchanged around 0.9 angstrom during the course of time evolution up to 8 picoseconds. Notice that for the $\mathrm{H}_{2}$ fragment to rotate 360 degrees, it visits three times of both the $C_{s}(\mathrm{I})$ and $C_{s}(\mathrm{II})$ conformers.

Time profiles of the frontier orbitals, chemical potential, hardness, and electrophilicity index of the protonated methane are shown in figures 4 and 5. It is seen that as the floating $\mathrm{H}_{2}$ rotates above the $\mathrm{CH}_{3}^{+}$ plane, these DFT concepts and indices fluctuate as well. One observation from HOMO/LUMO time profiles (shown in the left panel of figure 4) is that HOMO fluctuates more widely and thus illustrates a more dynamic behavior than LUMO. On the other hand, a peak in the hardness profile represents a local minimum in the conformation space as a larger HOMO-LUMO gap stands for a stable conformer according to the maximum hardness principle, whereas a valley (and thus smaller HOMO-LUMO

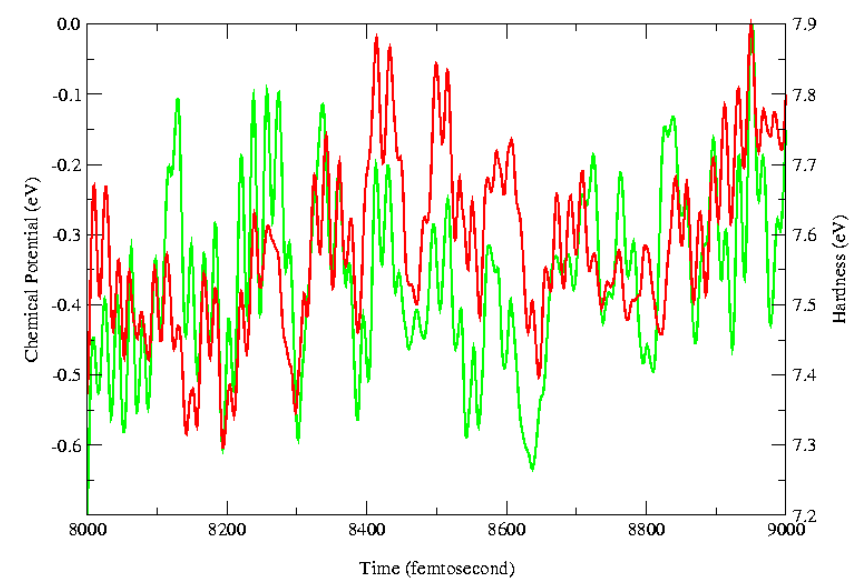

Figure 8. Comparison of a portion of QMD time profiles of chemical potential (green, lower curve) and global hardness (red, upper curve) during the conformation change of the $\mathrm{Cl}^{-}$ion in 30 water molecules where the $\mathrm{Cl}^{-}$ion comes out from the interior to the surface of the water cluster. gap) in the hardness evolution denotes a transition between two local minima. In the chemical potential time profile, a similar pattern follows. That is, a peak represents a visit to a stable conformer and a valley stands for a transition. A portion of the time profile is shown in figure 5 to illustrate the positive correlation between the time evolution of the chemical potential and hardness, where we can see clearly that, though not always, a peak in hardness often corresponds to a peak in chemical potential and vice versa. The concurrent dynamic behavior of chemical potential and hardness comes from the fact that HOMO is more dynamic than LUMO, and thus the former governs the dynamic behavior of $\mu$ and $\eta$ via (5) and (6). These results demonstrate that chemical potential and hardness change accordingly and coherently as molecular conformation alters, indicating that the DFT concepts, long employed merely as chemical reactivity indices, can also be used to serve as good indicators for conformation changes. Note that the frequency of $\mu$ and $\eta$ alternations is faster than the $\mathrm{H}_{1}-\mathrm{H}_{2}-\mathrm{C}-\mathrm{H}_{3}$ dihedral angle change in figure 3 . This is because, as noted above, each time the dihedral angle rotates a cycle, it passes three times of the two local minima, $C_{s}(\mathrm{I})$ and $C_{s}(\mathrm{II})$, and thus $\mu$ and $\eta$ changes should be three times faster than the dihedral angle alternation. Also shown in figure 4 (right panel) is the dynamic behavior of the electrophilicity index, where one finds substantially different value of $\omega$ is likely during the course of time evolution implying that the electron-acquiring capability of an electrophile can be different by a fact of 3 or more.

In the second example, we study a chloride anion soaked in 30 water molecules, the $\mathrm{Cl}^{-}\left(\mathrm{H}_{2} \mathrm{O}\right)_{30}$ cluster. The starting conformation of our simulation, as shown on the left hand side of figure 6 , is that the chloride anion is placed in the middle of the water cluster. This system has been investigated before in the literature from the viewpoint of classical and polarizable force-field molecular dynamics, ${ }^{28,29}$ Monte Carlo simulations, ${ }^{30,31}$ etc., where one finds that the chloride ion tends to surge from the interior to the surface, leading to the energetically more favorable conformation, as shown in the right picture of figure 6. Our QMD simulation correctly grasps such a dynamic feature, observing that the ion emerges from the interior in less than 2 picoseconds, contrasting to $\sim 100$ ps what polarizable force-field MD simulations have observed, ${ }^{28,29}$ indicating that QMD simulations are able to demonstrate the feature in a much shorter simulation time. Figure 7 shows the time pro 

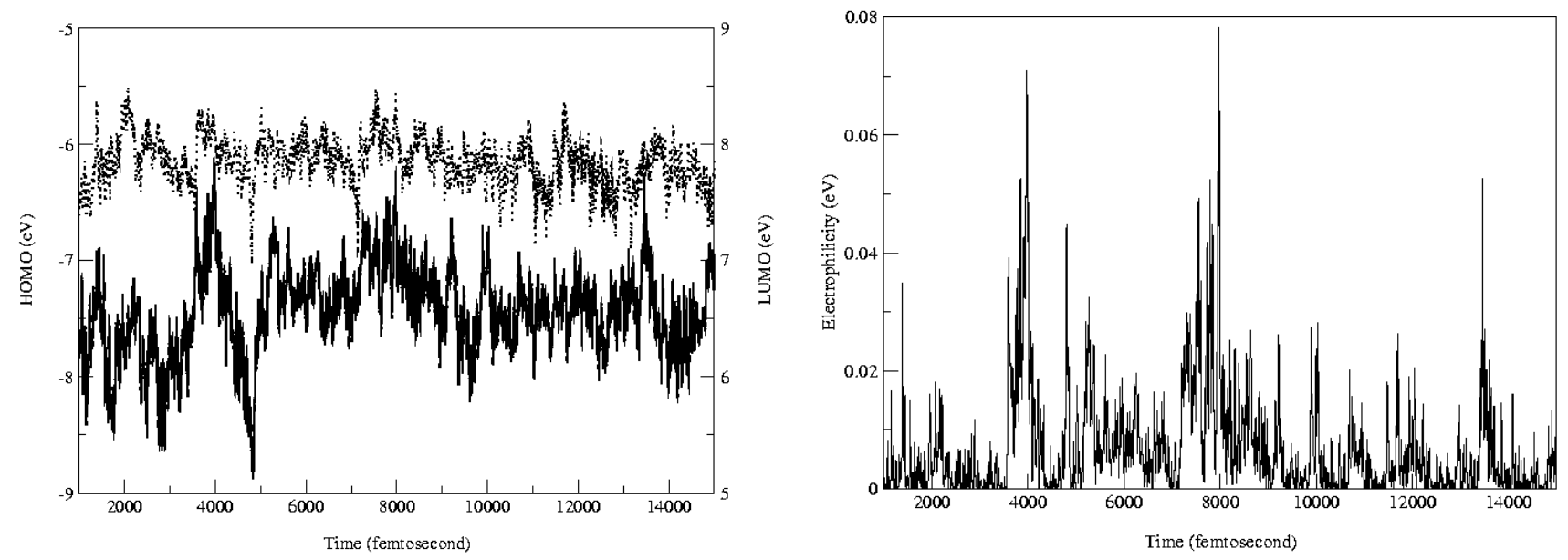

Figure 9. Profiles of HOMO (solid line, left panel) and LUMO (dotted line, left panel) and electrophilicity index (right panel) for the $\mathrm{Cl}^{-}$ion in 30 water molecules, $\mathrm{Cl}^{-}\left(\mathrm{H}_{2} \mathrm{O}\right)_{30}$.
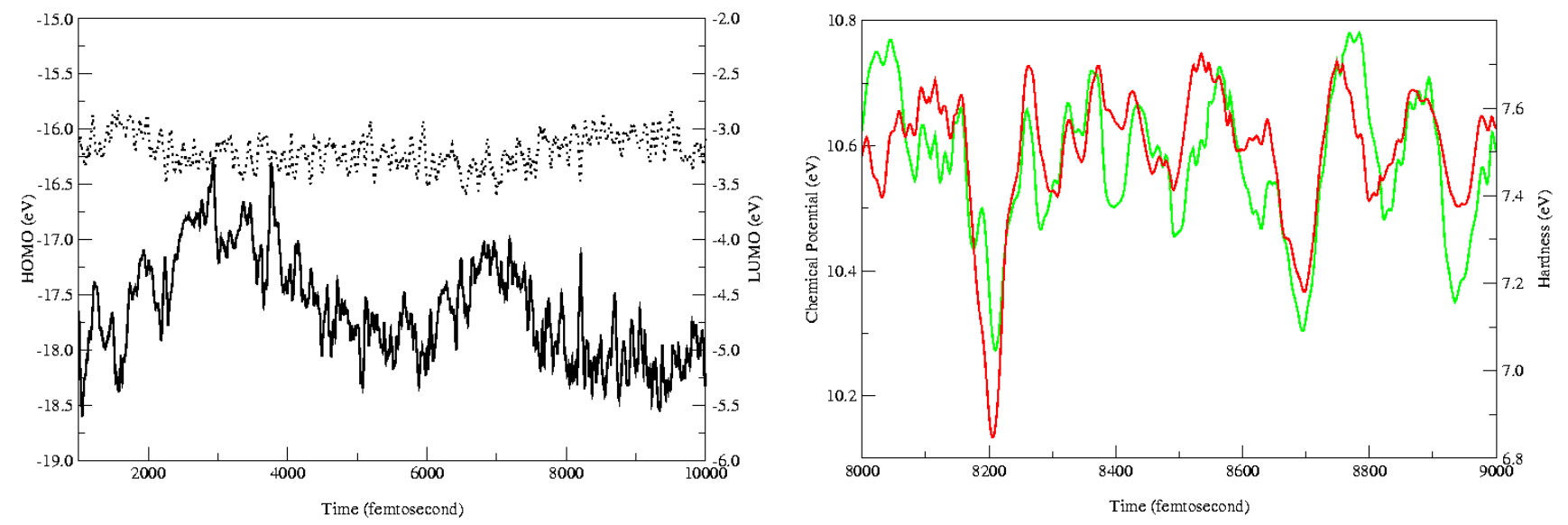

Figure 10. Time evolution of HOMO (solid line, left) and LUMO (dotted line, left) energies and chemical potential (blue line, right) and hardness (red line, right) for the $\mathrm{Ca}^{2+}\left(\mathrm{H}_{2} \mathrm{O}\right)_{15}$ cluster.

file of the total SCF energy evolution of the system and the associated hardness profile, in which it is seen that the process is energy decreasing and thus enthalpy driven. From the hardness profile, one finds that as the conformation of the system changes from the less stable conformer where $\mathrm{Cl}^{-}$stays inside the water cluster to the energetically more favorable state with $\mathrm{Cl}^{-}$coming to the surface to the water cluster, hardness increases considerably, conforming to the notion that a stable structure possesses a bigger HOMO-LUMO gap. Plotting the chemical potential and hardness profiles together, in figure 8 , we confirm what has been seen in the $\mathrm{CH}_{5}^{+}$case, where these two DFT reactivity indices often behave concurrently - a $\eta$ peak also usually corresponds to a $\mu$ peak and vice versa. This coherence of dynamic behavior between chemical potential and hardness again comes from the fact that HOMO is more dynamic than LUMO, whose profiles are plotted in figure 9 together with the very viable electrophilicity index.

Our last example is for the $\mathrm{Ca}^{2+}\left(\mathrm{H}_{2} \mathrm{O}\right)_{15}$ cluster, where $\mathrm{Ca}^{2+}$ ion sits in the middle of 15 water molecules. Figure 10 shows the profiles of HOMO/LUMO (left panel) and chemical potential and hardness (right picture), confirming again that HOMO possesses 
more dynamic behavior than LUMO and that $\mu$ and $\eta$ often fluctuate concurrently, a $\eta$ peak also usually corresponds to a $\mu$ peak and vice versa.

\section{Concluding remarks}

DFT concepts, indices and rules are well established, but have mainly been understood and applied in the literature in the context of chemical reactivity and in the time-independent domain. In this work, we make use of the Bohn-Oppenheimer quantum molecular dynamics and investigate the dynamic behavior of some of these concepts such as frontier orbitals, chemical potential, hardness, and electrophilicity index in association with conformation changes of a few small to medium-sized molecular systems, demonstrating that these theoretically sound entities can also be employed to understand dynamic processes such as conformation changes as well as other dynamic phenomena alike. More pursuits along this line are in process.

\section{References}

1. Parr R G and Yang W 1989 Density functional theory of atoms and molecules (New York: Oxford University Press)

2. Geerlings P, De Proft F and Langenaeker W 2003 Chem. Rev. 1031793

3. Parr R G, Von Szentpaly L and Liu S 1999 J. Am. Chem. Soc. 1211922

4. De Proft F and Geerlings P 2001 Chem. Rev. 1011451

5. Chattaraj P K and Roy D R J. Phys. Chem. A (in press)

6. Chattaraj P K 1992 Int. J. Quantum Chem. 41845

7. Chattaraj P K and Nath S 1994 Int. J. Quantum Chem. 49705

8. Chattaraj P K and Nath S 1994 Chem. Phys. Lett. 217 342
9. Chattaraj P K and Sengupta S 1996 J. Phys. Chem. 10016126

10. Chattaraj P K and Sengupta S 1997 J. Phys. Chem. A101 7893

11. Chattaraj P K and Maiti B 2001 J. Phys. Chem. 105 169

12. Chattaraj P K and Maiti B 2004 J. Phys. Chem. 108 658

13. Vuilleumier R and Sprik M 2001 J. Chem. Phys. 115 3454

14. Car R and Parinello M 1985 Phys. Rev. Lett. 552471

15. York D M and Yang W 1996 J. Chem. Phys. 104 159

16. Bolton K, Hase W L and Peshlherbe G H 1998 Modern methods for multidimensional dynamics computation in chemistry (ed.) D L Thompson (Singapore: World Scientific) p. 143

17. Aprà E et al 2005 NWChem. A computational chemistry package for parallel computers version 47, Pacific Northwest National Laboratory, Richland, Washington 99352-0999, USA

18. Kendall R A et al 2000 Comput. Phys. Commun. 128 260

19. Parr R G, Donnelly R A, Levy M and Palke W E 1978 J. Chem. Phys. 683801

20. Mulliken R S 1934 J. Chem. Phys. 2782

21. Parr R G and Pearson R G 1983 J. Am. Chem. Soc. 1057512

22. Koopmans T A 1933 Physica 1104

23. Parr R G, Von Szentpaly L and Liu S 1999 J. Am. Chem. Soc. 1211922

24. Gerlich D 2005 Chem. Phys. Phys. Chem. 71583

25. Brown A, Mccoy A B, Braams B J, Jin Z and Bowman J M 2004 J. Chem. Phys. 1214105

26. Mccoy A B, Braams B J, Brown A, Huang X C, Jin Z and Bowman J M 2004 J. Phys. Chem. A108 4991

27. Schreiner P R 2000 Angew. Chem. Int. Ed. 393239

28. White E T, Tang J and Oka T 1999 Science 284135

29. Herce D H, Perera L, Darden T A and Sagui C 2005 J. Chem. Phys. 122024513

30. Markovich G, Perera L, Berkowitz M L and Cheshnovsky O 1996 J. Chem. Phys. 1052675

31. Shevkunov S V, Lukyanov S I, Leyssale J M and Millot C 2005 Chem. Phys. 31097 